\title{
Patient Survey of the Physical, Emotional, and Informational Challenges for Patients Living with Neuroendocrine Tumors
}

\author{
Mohid S. Khan · Katharina Mellar · Rupert Watts · Isabelle Bocher-Pianka • \\ Abdelali Majdi · Grace Goldstein
}

Received: September 23, 2019 / Published online: December 2, 2019

(C) The Author(s) 2019

\section{ABSTRACT}

Introduction: Patient surveys of disease burden on daily living rarely explore the impact that patient information sources can have on optimizing care.

Methods: To identify unmet needs/possible solutions for information and support to

Katharina Mellar died in August 2018.

Enhanced Digital Features To view enhanced digital features for this article go to https://doi.org/10.6084/ m9.figshare.10272449.

Electronic Supplementary Material The online version of this article (https://doi.org/10.1007/s40487019-00103-2) contains supplementary material, which is available to authorized users.

M. S. Khan $(\bowtie)$

Department of Gastroenterology, University

Hospital of Wales, Cardiff CF14 4XW, UK

e-mail: khanms14@cardiff.ac.uk

K. Mellar

Netzwerk Neuroendokrine Tumoren (NeT) e.V., Nuremberg, Germany

R. Watts

Kanga Health Ltd, Meadowside, Mountbatten Way, Congleton, UK

I. Bocher-Pianka · A. Majdi

Ipsen, Boulogne Billancourt, France

G. Goldstein

The Carcinoid Cancer Foundation, White Plains,

NY, USA address the physical, emotional, and informational challenges that patients living with neuroendocrine tumors (NETs) may have, an online survey was conducted among patients recruited by the Carcinoid Cancer Foundation (US), Netzwerk Neuroendokrine Tumoren (NeT) e.V. (Germany), and Association de Patients porteurs de Tumeurs Endocrines Diverses (France); these organizations approved the survey questions.

Results: Between August 2015 and November 2015, 741 online surveys were completed in France $(n=73)$, Germany $(n=240)$, and the US $(n=428)$ during a 6 -week period. Over $65 \%$ of patients were diagnosed $\leq 5$ years ago. Across the three countries, multiple symptoms were experienced before diagnosis. These symptoms were consistent with those of functional tumors, most commonly diarrhea (40-47\%) and flushing (12-45\%). The most common emotion upon diagnosis was anxiety (20-67\%). The greatest physical challenges for patients living with NETs included fatigue (26-66\%), diarrhea (22-48\%), pain/discomfort (13-40\%), and sleep disturbance (16-35\%), despite cancer management. Information sources classed as "very useful" included patient-association websites, disease awareness websites, and journals; "patients-like-me" case studies were most favored $(43-67 \%)$ for future use as sources of information.

Conclusion: Patients with NETs face negative emotions and multiple physical challenges that 
require psychological support/coping strategies, and they seek reliable, personally relevant, easily understood information, including that from interactions with other patients.

Plain Language Summary: Plain language summary available for this article.

Keywords: Emotional challenges; Neuroendocrine tumors; Patient information; Patient survey; Physical challenges

\section{Key Summary Points}

Why carry out this study?

A survey was developed to identify the usefulness of information sources for patients (in Germany, France, and the US) with neuroendocrine tumors (NETs) - an area that is not often explored.

The survey aimed to understand the physical, informational, and emotional challenges that are faced before, at, and after the diagnosis of a NET; identify key challenges that are faced by patients; understand where patients obtain information and support; and determine whether they are able to find the information needed.

\section{What was learned from the study?}

Patients living with NETs use a variety of information sources that vary in availability and popularity between countries.

Respondents valued resources and support from other patients with NETs, such as direct contact and interaction as well as "patients-like-me" case studies.

The variety of responses indicate that a "one-size-fits-all" approach is not appropriate in NETs, reflecting the diverse nature of the condition and individual preferences, and that a tailored approach to symptom management, information, and support is needed.

\section{PLAIN LANGUAGE SUMMARY}

An online survey was developed to identify the usefulness of information available for patients with neuroendocrine tumors, also called NETs. The survey had 12 questions with multiplechoice answers or free-text options. The survey aimed to:

- Understand the physical, informational, and emotional challenges faced before, at, and after the diagnosis of NET

- Identify key challenges faced by patients

- Understand where patients obtain information and support, and whether they are able to find the information needed.

The survey was sent to patients with NETs by three patient or patient advocate organizations. There were 428 respondents in the United States, 240 in Germany, and 73 in France.

The survey found that the most common emotion when people received their diagnosis was anxiety. The biggest physical challenges were fatigue and diarrhea. Findings regarding the best sources of information and the easiest way to understand information varied among the countries. Information sought and not found by NETs patients included:

- Information about treatments

- Causes of the tumors

- Prognosis

- Nutritional advice

- Psychological support

- The opportunity to discuss their experiences with other patients with NETs.

Key findings from this survey were:

- Patients living with NETs use a range of information sources, which vary between countries in their availability and popularity.

- Respondents valued resources and support from other patients with NETs, such as direct contact and interaction as well as "patientslike-me" case studies.

- The variety of responses indicate that a tailored approach to symptom management, information, and support is needed, reflecting the diverse nature of the condition. 


\section{INTRODUCTION}

In recent years, frameworks have been developed to assist with decisions on access to treatment; however, such frameworks tend to attach greater importance to the clinically focused values of healthcare professionals than to outcomes that also matter to patients [1]. In recognition of the importance of connecting patient experience with clinicians' understanding, patient-reported outcomes (PROs) are becoming increasingly popular, as described in a recent systematic review by Yang et al. While it has been suggested that analyzing data from standardized PROs can improve patient-doctor dialog, symptom management, care satisfaction, and quality of life [2], further research is needed to understand how much the use of PROs can affect symptom awareness and patient outcomes. The authors also highlight the need for PROs to appropriately assess issues that are relevant to patients [2].

Insights from patient surveys of disease burden and the impact on daily living may be key to optimizing care, but it is also important for such surveys to address the emotional impact of a condition, in addition to the physical challenges a condition may pose, to reflect the patient experience. Neuroendocrine tumors (NETs) are increasingly prevalent, though uncommon, cancers that are heterogeneous in origin and behavior. With a varied survival, care of NETs patients requires the management of a cancer and chronic disease [3]. Although a variety of patient resources have been developed over the last decade by several treatment centers and organizations across different countries, the usefulness of information sources used by patients living with NETs is rarely explored.

In 2016, data were published from the first global (more than 12 countries), self-reported survey of 1928 patients with NETs, conducted under the auspices of the International Neuroendocrine Cancer Alliance (INCA) and Novartis $[4,5]$. The report on this qualitative survey includes a comprehensive and concise summary of the range of issues faced by patients with NETs, including challenges in obtaining a diagnosis, the wide range of symptoms experienced, limited treatment options, scarcity of disease information, and impact on personal and work life, emotional well-being, and relationships. The report highlights the unmet needs of patients with NETs from their own perspective, in particular their need for NETspecific treatments and information [4].

To identify unmet patient needs and possible solutions for an online patient information and support resource that would address the physical, emotional, and informational challenges patients with NETs may have, a three-country patient survey was conducted. Specifically, this survey aimed to facilitate a greater understanding of (1) the physical, informational, and emotional challenges facing patients before, at, and after their diagnosis of NET and (2) where patients obtain information and support to deal with these challenges, the channels they use, and whether they can find the information they need.

\section{METHODS}

\section{Patients, Patient/Patient Advocate Organizations, and Survey Dissemination}

An online patient survey (conducted via the SurveyMonkey platform) was developed to gather patient insights to support the development of an online information resource for patients living with NETs. The survey was disseminated to individuals with NETs by three patient/patient advocate organizations-L'Association des Patients porteurs de Tumeurs Endocrines Diverses (APTED, https://www. apted.fr/) in France, Netzwerk Neuroendokrine Tumoren (NeT) e.V. (https://www.glandula-netonline.de) in Germany, and the Carcinoid Cancer Foundation (CCF, https://www. carcinoid.org) in the US-via news articles and banners on their websites, social media posts and tweets, email newsletters, and emails. The survey was disseminated to individuals who were members of the patient/patient advocate organizations and to nonmembers of these organizations. The survey was conducted over the following periods in 2015: October 15 to November 9 (France), September 1 to September 
30 (Germany), and August 11 to September 2 (US).

\section{Compliance with Ethics Guidelines}

Respondents were aware of the purpose of the survey and gave their permission for their insights to be gathered in this voluntary process. Owing to the nature of the research as an insight-gathering exercise rather than as a clinical-grade piece of research, and the recruitment methods used, approval by an independent ethical committee was not required for this survey.

\section{Survey Questions}

The survey comprised the 12 questions listed in Table 1. One additional question was provided to the US respondents, who were asked about the type of primary NET they had (question 2). Survey questions were drafted, and all were then checked and approved by the respective patient/patient advocate organizations and phrased in French, English, or German, depending on the target country. To ensure that each question within the survey was appropriate for the supporter base/membership of each of the patient/patient advocate organizations, questions were edited slightly in consultation with the respective groups. Therefore, there were some minor between-country variations in question phraseology. Most questions had multiple-choice answers, including options for free-text entry ("other"), and two questions were answered with rating scales, allowing additional information to be captured. There

Table 1 Survey questions

\begin{tabular}{|c|c|c|}
\hline $\begin{array}{l}\text { Question } \\
\text { number }\end{array}$ & Question & Notes \\
\hline 1 & $\begin{array}{l}\text { How long ago were you diagnosed with carcinoid cancer or another } \\
\text { neuroendocrine tumor }(\mathrm{NET}) \text { ? }\end{array}$ & \\
\hline 2 & What type of primary carcinoid cancer or NET do you have? & US survey only \\
\hline 3 & Who diagnosed you? & \\
\hline 4 & Which tests or diagnostics were performed? & \\
\hline 5 & $\begin{array}{l}\text { Which of the following symptoms did you have prior to receiving your } \\
\text { diagnosis? }\end{array}$ & \\
\hline 6 & How did you feel after your diagnosis? & \\
\hline 7 & What are your biggest physical challenges that your condition poses you? & \\
\hline 8 & After your diagnosis where did you go for information? & \\
\hline 9 & $\begin{array}{l}\text { How useful was the information you found in each of these channels } \\
\text { (if applicable)? }\end{array}$ & $\begin{array}{l}\text { Rating scale for specified } \\
\text { options }\end{array}$ \\
\hline 10 & $\begin{array}{l}\text { How easy to understand was the information you found in each of these } \\
\text { channels (if applicable)? }\end{array}$ & $\begin{array}{l}\text { Rating scale for specified } \\
\text { options }\end{array}$ \\
\hline 11 & What information didn't you find that you really needed? & $\begin{array}{l}\text { Answers to this question were } \\
\text { free text only }\end{array}$ \\
\hline 12 & What formats would you like to receive information in? & \\
\hline 13 & Are there any other thoughts or suggestions you would like to make? & $\begin{array}{l}\text { Answers to this question were } \\
\text { free text only }\end{array}$ \\
\hline
\end{tabular}

Unless otherwise stated, questions were included on all versions of the questionnaire and were multiple choice answers 
were variations in answer options between countries.

\section{Survey Analysis}

Results from respondents in the three countries were collated for qualitative evaluation, including free-text responses and example responses, which are presented herein. RW analyzed the data from the questionnaires, including free-text responses; no formal statistical analyses were performed on these data.

\section{RESULTS}

A total of 741 patients completed online surveys: France $(n=73)$, Germany $(n=240)$, and the US $(n=428)$. A diagnosis of NET had been made up to 5 years before the survey period in $66 \%$ of respondents: specifically, $76 \%(n=53 /$ 70 respondents) in France, 64\% $(n=150 / 233$ respondents) in Germany, and 66\% $(n=268$ / 406 respondents) in the US. Overall, the largest proportion of patients (31-41\%) were diagnosed 3-5 years before the survey period (Fig. 1). The type of primary NET as reported by patients at diagnosis was recorded for participants in the US (see Fig. S1 in the Electronic supplementary material, ESM).

Before diagnosis, patients often experienced multiple symptoms; these were generally gastrointestinal symptoms combined with other symptoms, which may reflect those of carcinoid syndrome in a subgroup of patients. Among these symptoms, the most common were diarrhea, flushing, bowel or bladder habit changes (including incontinence), and persistent pain (Fig. 2a). "Other" symptoms included chest/abdominal pain, wheezing and breathing difficulties, edema, nausea/vomiting, and anemia.

Overall, anxiety was the predominant emotion after diagnosis, with many patients feeling overwhelmed by the implications of their diagnosis; however, many patients reported feeling resigned (Fig. 2b). The proportion of participants who said they felt confused or perplexed varied widely between countries (5.7\% in France, $21.2 \%$ in Germany, and 39.3\% in the US). A relatively small proportion of a France ( $n=70$ respondents)

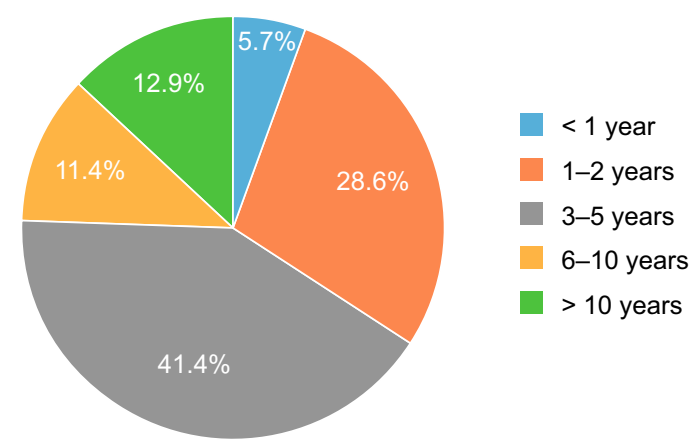

b Germany ( $n=233$ respondents)

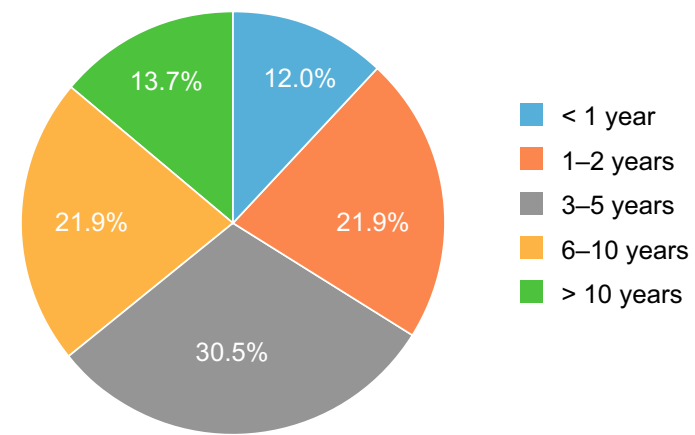

C US ( $n=406$ respondents)

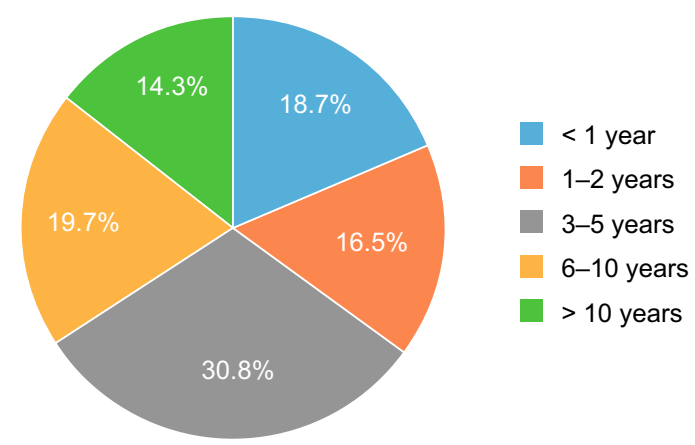

Fig. 1 Time between NET diagnosis and survey completion: proportion of respondents by each time period between NET diagnosis and survey completion in a France, $\mathbf{b}$ Germany, and $\mathbf{c}$ the US. NET neuroendocrine tumor

patients felt empowered by their diagnosis $(4.4 \%$ felt energized in France, $2.3 \%$ felt encouraged, empowered, or strengthened in Germany, and $4.5 \%$ felt empowered in the US). A distinctly larger proportion reported relief upon being diagnosed (8.9\% in France, 14.4\% in Germany, and $18.4 \%$ in the US) (Fig. 2b). 


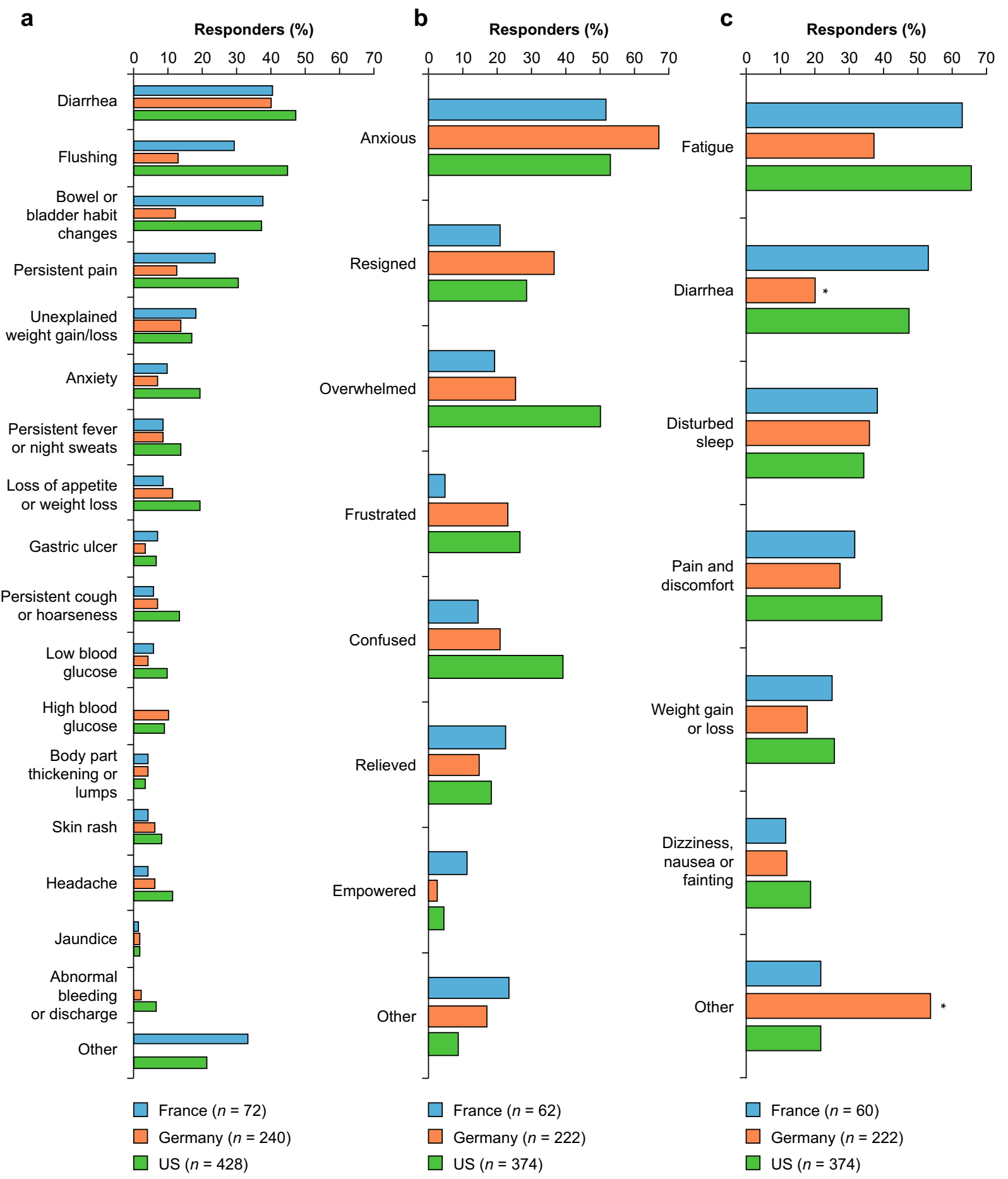


4Fig. 2 a Symptoms experienced before receiving NET diagnosis; $\mathbf{b}$ emotions experienced after receiving NET diagnosis; c physical challenges of living with NETs. Proportions of respondents who experienced each challenge in France, Germany, and the US are shown. In a, "other" symptoms include pain, vomiting, and shortness of breath. In b, "other" includes feeling sad, angry, energized, shocked, fearful, and afraid. In c, "other" includes anxiety and diarrhea following the operation. ${ }^{*}$ No German patients specifically selected "diarrhea," but $20 \%$ of those who selected "other" stated that they had diarrhea after an operation. It is unknown whether they experienced diarrhea beforehand. Diarrhea could be caused by consequences of surgery and treatments rather than the disease. NET neuroendocrine tumor

Patients living with NETs reported fatigue, diarrhea, sleep disturbance, and pain and discomfort as their greatest physical challenges (Fig. 2c).

Overall, the diagnosis of NET was most frequently made by a gastroenterologist, particularly in France (73\%) and the US (30\%) (Fig. 3). Of the 124 German patients who selected "other," 44 (35\%) noted that they had been diagnosed during surgery, whereas three (23\%) of the 13 French patients who selected "other" reported that they had been diagnosed by a radiologist. Computed tomography (CT) or computerized axial tomography (CAT) scans were the most frequently used diagnostic modalities across the three countries (74-80\%), followed by blood tests (59-68\%), biopsies (59-62\%), and urinary 5-hydroxyindoleacetic acid testing (36-68\%) (Table 2). German patients tended to report having undergone the broadest range and the greatest number of tests and diagnostic examinations. Many German patients who selected "other" noted that they had a colonoscopy or an ultrasound examination. "Endoscopy" but not "colonoscopy" was listed as an optional answer in France, so some French patients who had colonoscopies may have selected "endoscopy."

A high degree of variability in approaches to seeking information was observed across patients from France, Germany, and the US (Fig. 4). The Internet and search engines were most frequently used overall. Patient- a France $(n=62)$

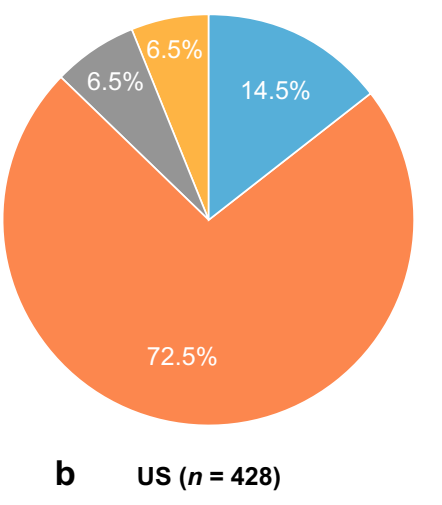

Endocrinologist

Gastroenterologist

Oncologist

Pulmonologist

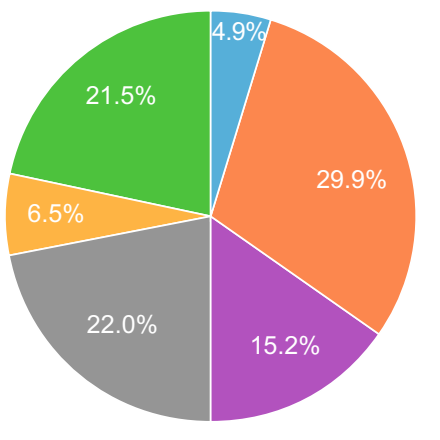

Endocrinologist

Gastroenterologist

GP

Oncologist

Pulmonologist

Other

C Germany $(n=240)$

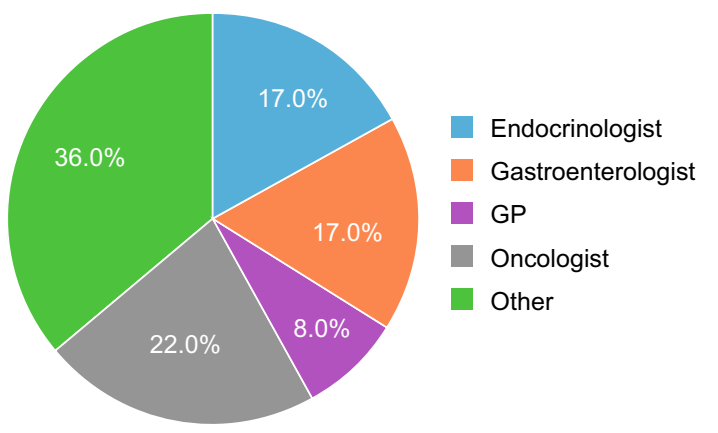

Fig. 3 Physicians who made the diagnosis of primary carcinoid cancer or neuroendocrine tumor: proportions of respondents in a France, $\mathbf{b}$ the US, and $\mathbf{c}$ Germany. Other includes radiologist, pathologist, surgeon, and nurse practitioner

association websites and patient brochures or information booklets were most commonly used by French and German patients. NET/carcinoid disease awareness websites and support groups as well as general cancer websites were used most often by patients in the US. In all three countries, family and friends also provided information from unspecified sources to 
Table 2 Tests or examinations performed during diagnosis: proportions of respondents who reported that they underwent each test in France, Germany, and the US

\begin{tabular}{|c|c|c|c|}
\hline \multirow[t]{2}{*}{ Test/examination } & \multicolumn{3}{|c|}{ Proportion of patients who reported that they underwent each test, $n(\%)$} \\
\hline & France $(n=70)$ & Germany $(n=240)$ & US $(n=428)$ \\
\hline 5-HIAA urine test & $25(35.7)$ & $163(67.9)$ & $206(48.1)$ \\
\hline Biopsy & $41(58.6)$ & $147(61.3)$ & $256(59.8)$ \\
\hline Blood tests & $43(61.4)$ & $163(67.9)$ & $253(59.1)$ \\
\hline Colonoscopy $^{\mathrm{a}}$ & $0(0)$ & $0(0)$ & $183(42.8)$ \\
\hline CT/CAT scan & $52(74.3)$ & $167(69.6)$ & $344(80.4)$ \\
\hline Endoscopy & $16(22.9)$ & $0(0)$ & $154(36.0)$ \\
\hline MRI scan & $29(41.4)$ & $118(49.2)$ & $163(38.1)$ \\
\hline Physical examination & $6(8.6)$ & $165(69.8)$ & $169(39.5)$ \\
\hline $\mathrm{X}$-ray & $12(17.1)$ & $99(41.3)$ & $108(25.2)$ \\
\hline Other & $20(28.6)$ & $61(25.4)$ & $90(21.0)$ \\
\hline
\end{tabular}

5-HIAA 5-hydroxyindoleacetic acid, CAT computerized axial tomography, CT computed tomography, MRI magnetic resonance imaging

${ }^{a}$ Colonoscopy was not included as a country-specific test for France

patients (reported by $3.2 \%$ of respondents in France, $23.4 \%$ of respondents in Germany, and $21.1 \%$ of respondents in the US). Interestingly, relatively few patients used social media to seek information (Fig. 4).

Different information sources were valued by patients in the three countries. In France, the information sources regarded by patients as being the most useful included patient-association websites, government/health-authority websites, and brochures (see Fig. S2a in the ESM); in Germany, specialist NET journals, patient-association websites, Internet searches, and information booklets were rated as the most useful resources (see Fig. S2b in the ESM). In the US, patients reported NET/carcinoid disease awareness websites, NET/carcinoid support groups, and general cancer websites to be the most useful resources (see Fig. S2c in the ESM). It should be noted that, in Germany, select journals specializing in NET information, patient booklets, and patient-association websites are edited and published by Netzwerk NeT e.V.
In France, the sources of information that were considered the easiest to understand were patient-association websites and brochures (see Fig. S3a in the ESM), whereas in Germany, family and friends were considered the easiest information source to understand, followed by Internet searches and websites or self-help organizations (see Fig. S3b in the ESM). In the US, NET/carcinoid disease awareness websites and NET/carcinoid support groups were considered to provide the most easily understandable information (see Fig. S3c in the ESM). Despite being regarded as one of the most useful information sources, health-authority websites were generally not found to be very easy to understand by patients [3/34 patients in France $(8.8 \%) ; 14 / 87$ patients in Germany $(16.1 \%)$; $25 / 147$ patients in the US (17.0\%)].

In general, the free-text responses given to questions about the information on NETs sought by patients (Figs. 5, 7) and their preferred information format (Fig. 6) suggest an unmet need for simple, clear, precise, and focused explanations that are readily accessible, not too technical, and relevant to 


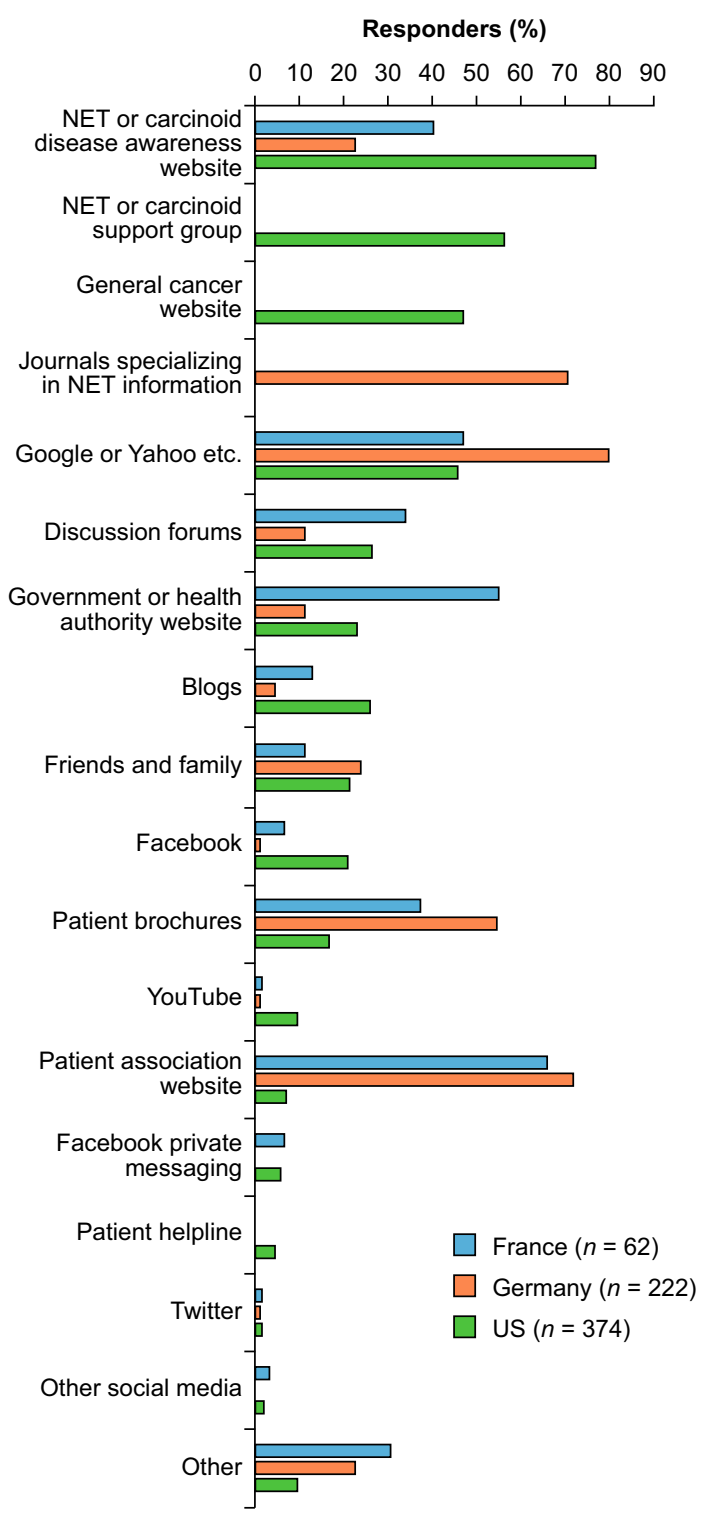

Fig. 4 Sources used by patients to seek information about NETs: proportions of respondents who used each source in France, Germany, and the US. Other includes general practitioner, oncologist, and family expert. NET neuroendocrine tumor

circumstantial, physical, and/or emotional difficulties. Participants reported having unanswered questions about the disease, including its causes, progression, and prognosis, treatment options, and whether lifestyle choices such as nutrition may impact the disease. Patients also highlighted their need for psychological support.
Overall, "patients-like-me" case studies were the most favored format in which to receive information in France (89.1\% of respondents) and the US (66.8\% of respondents), and were also favored in Germany (62.2\% of respondents) (Fig. 6). Patients in Germany preferred opportunities to "discuss with experts" (82.4\%) and disease-specific booklets or brochures (80.2\%). Emails and e-newsletters were other formats preferred by patients in the US.

Free-text responses that gave additional thoughts or suggestions from survey respondents revealed a need for improved accessibility to easy-to-understand patient information, and for support. In addition, the need to increase awareness and understanding of NETs among non-NET specialist physicians was highlighted, with six requests submitted for more physician education about this rare condition. In particular, disease education was requested for the wider medical community, including nonspecialists and nononcologists, such as that already provided by some patient/patient advocate associations, for example Netzwerk NeT e.V. in Germany (Fig. 7).

\section{DISCUSSION}

Qualitative evaluation of this patient survey performed in France, Germany, and the US shows that a high proportion of patients living with NETs experience ongoing physical symptoms (most commonly fatigue, diarrhea, sleep disturbances, and pain and discomfort), despite managing their cancer. This is a large international survey of 741 patients with a relatively uncommon cancer, and it highlights a need for better management of the causes of diarrhea, pain, fatigue, and sleep disturbance by physicians managing NETs, research in these areas, and the development of support services involving multidisciplinary teams (e.g., gastroenterology, pain teams, psychology/counseling, and physiotherapy). A summary of the key messages and results of the survey can be found in Fig. S4 in the ESM.

The physical and emotional challenges of patients with NETs are augmented by the long diagnostic journey they experience, as noted in 


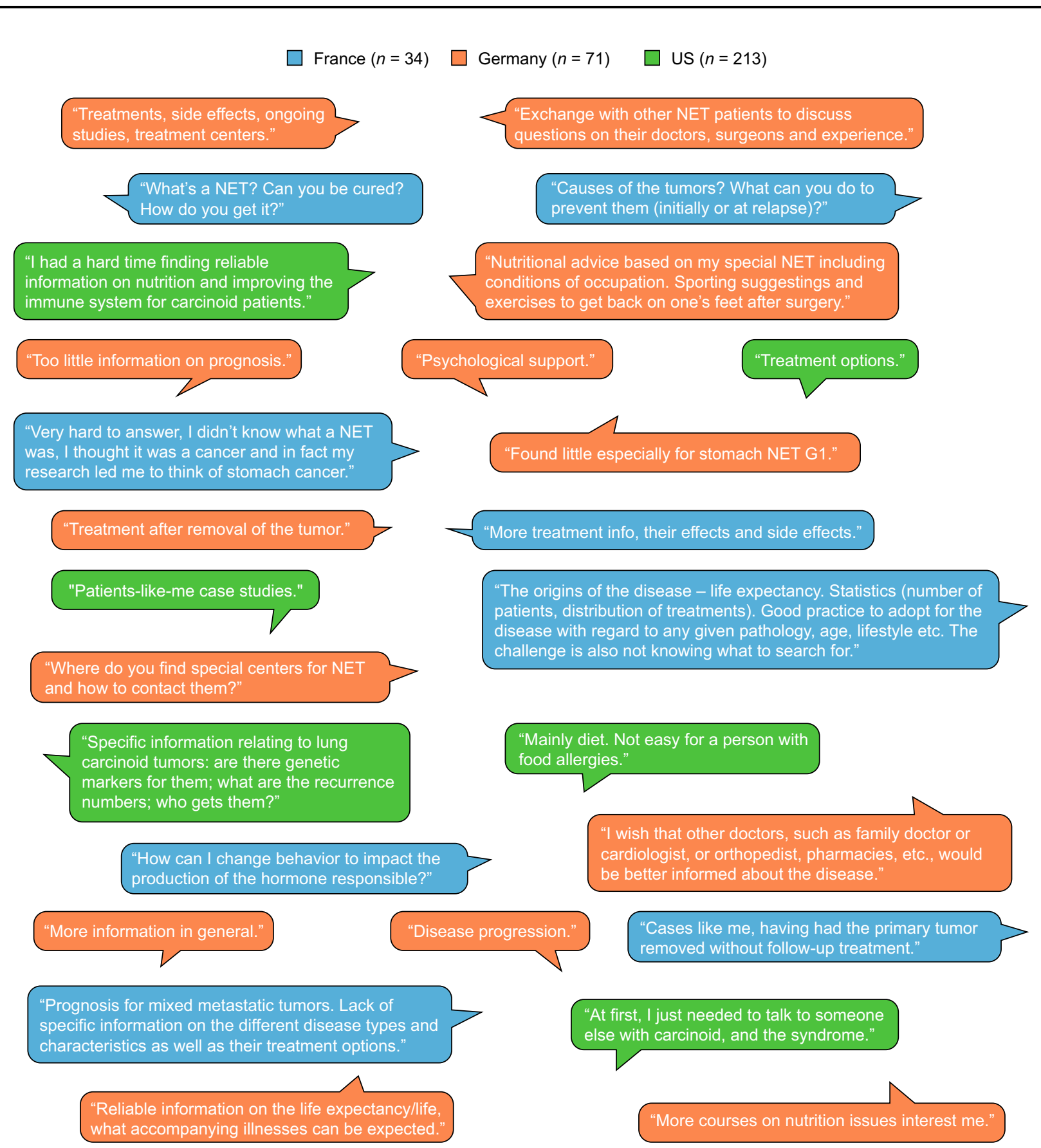

Fig. 5 Examples of information sought, but not found, by participants. NET neuroendocrine tumor

previous studies $[4,5]$. Many patients with this disease reported experiencing negative emotions such as anxiety, fear, feeling overwhelmed, and confusion after their diagnosis. Acceptance of their diagnosis was characterized by a sense of empowerment or relief in some patients, possibly because diagnosis allowed them to understand their condition and to start to take control of its management. Our survey could not consider the variety of different personalities, education, and cultural background of patients, and it is acknowledged that people react in different ways. 


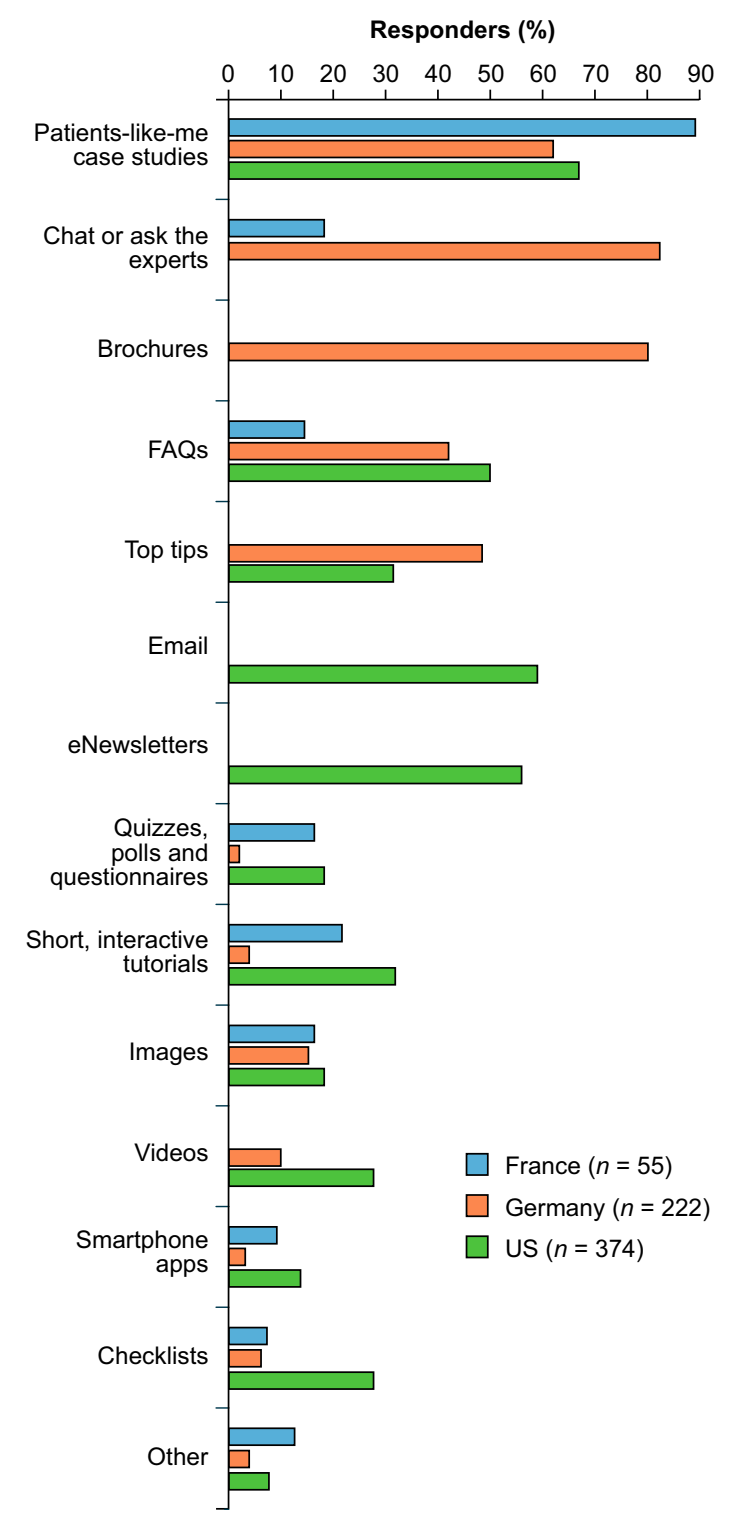

Fig. 6 Favored information formats: proportion of participants who favored each format in France, Germany, and the US. Other includes Internet sources and PDF/downloadable guides. FAQ frequently asked question

As well as revealing the needs of patients for psychological support and coping strategies around the time of diagnosis, these survey results also indicate that providing accessible and understandable information throughout the patient journey may help to address their emotional challenges. Gaining a deeper understanding of the long-term perspective of patients with NETs is expected to improve the development and focus of resources and support available to them.

The results of this survey, which are summarized in Fig. S4 in the ESM, describe both the physical and emotional challenges of living with NETs. In addition, the prevalence of confusion among survey respondents shows that they have unmet needs for information about their condition. This lack of disease information may contribute to the other negative emotions associated with the condition, exacerbated by an often-lengthy diagnosis route and uncertainty concerning the future behavior of the tumor. This survey adds to the findings of the 2016 INCA/Novartis survey $[4,5]$ by providing details on the information sources used that were useful and easy to understand by patients, on patients' preferred information formats, and on the information patients wanted but were unable to find.

Individuals living with NETs seek reliable, accessible, and easily understandable information that is relevant to their personal physical and psychological challenges [4]. Providing easy access to information resources tailored to patients' preferences, questions, and understanding may have the potential to help patients deal with their negative emotions and participate in decisions about their treatment and long-term care. Indeed, the finding from this survey that information on health-authority websites was not easily understood by patients, possibly because the terminology used on such sites is difficult to understand in these countries, highlights a need for information that is suitable for the varied health-literacy levels of patients.

Insights from this survey into the experience of living with NETs reveal issues, beyond clinical measures, that affect the well-being of patients with this condition and indicate the treatment outcomes that these patients desire. This understanding may contribute to future assessments of the holistic value of treatments and may also aid physicians in involving patients in disease-management decisions to optimize care $[1,2]$.

This survey found that patients with NETs use a variety of information sources, which varied between countries in their availability 




"My insurance company holding my long-term insurance dropped me after 2 years stating I was cured! They have no clue what we go through daily even after surgeries."

"There should be some sort of 'network' of patients and doctors where if you are diagnosed and consent to it then your info can be given to the group and the new patient can get an email or phone call from a qualified individual. This would have saved my family quite a bit of pain." support after the revelation of the disease that was pretty brutal. There was no specialist HCP able to advise on

now to manage anxiety linked to the shock of the diagnosis.

in fact I faced a barren desert in terms of immediate follow-up.

"Specialist site with private areas for patients to share advice on taking medication, advice on good injection techniques and alternatives, still not easy when you want to take a trip to the Antarctic. Information on your rights. Access to medical advice on line (even non-specialist, just advice)... to have a reference source for links to more information."

"This cancer just needs more public attention in general. There are too many people suffering over a period of years because no-one thinks of this disease. Mine had spread to my liver before I was properly diagnosed so I will never be cured." information on the different stages of the disease

The reports are, in my opinion, too technical. They give little info on treatment suggestions e.g. nutrition. possible complications.

More support groups and more awareness of NETs in general.'

Generally, doctors spend too little time educating themselves.

To be kept updated on possible insulinoma treatments overseas despite the lack of patients.

"My battle has not been with this cancer, my battle has been fighting insurance company to allow me to see specialists that specialize in treating this cancer."

"I would hope that the many technical terms could be put in parentheses and translated into an understandable German, so I can understand it.'

Fig. 7 Examples of additional thoughts and suggestions from participants. CCF Carcinoid Cancer Foundation, $H C P$ healthcare professional, NET neuroendocrine tumor

and popularity. Patient-association websites were the most commonly used information source in France and Germany, whereas disease support groups and disease awareness websites were the most used sources in the US. The perception of patients regarding what constitutes a patient-association website, a NET/carcinoid support group, and a NET/carcinoid disease awareness website may well have overlapped, however. Family and friends also provided patients with information; although the exact resources used by family and friends were not reported, patient/patient advocate organizations play an important role in providing information to both patients and their families and friends. The survey respondents valued 
resources and support from other patients with NETs, such as direct contact and interaction as well as "patients-like-me" case studies. Interestingly, relatively few patients used social media as an information source. There is the possibility that social media may have acted as a signpost to other sources, but this remains to be explored (potentially in a few years' time to identify any changes or progress made). Differences between information sources sought may have been affected by differences in socioeconomic background, culture, healthcare system, and accessibility; these data were not captured in this survey. Differences may also be due to differences in the complexity of terminology used in different information sources. Equally, it was not known whether patients were treated in specialized centers.

This survey was designed with the aim of gathering patient insights to help identify unmet patient needs and possible solutions for an online patient information and support resource. Following the initial analysis of the findings, the dissemination of the survey results was considered by the authors to be of potential benefit to the patient and therapy area community, despite some limitations of the survey design and analysis. In light of this, it should be noted that the qualitative nature of the evaluation of this survey somewhat limits the wider interpretation and application of the findings. In addition, the differences between the questions posed, their wording, and the corresponding available answers in each country limit the extent to which the survey results can be compared between countries. Another limitation is that indirect sources of information, for example those used by family and friends, were not reported, which may reduce the apparent use of some resources. Although asking open questions (with free-text responses) allows patients' voices to be heard, it does not facilitate statistical analysis or the drawing of single conclusions. It should also be noted that a retrospective patient survey such as this introduces recall bias regarding the type of NET, time of diagnosis, and modality/personnel making the diagnosis. Additionally, survey questions similar to those previously published $[4,5]$ have not undergone full questionnaire validation. As with a number of findings, we acknowledge selection bias as a limitation when conducting patient surveys, and further studies minimizing this bias should be undertaken. Finally, differences in healthcare personnel making the diagnoses may reflect different healthcare systems across the countries.

\section{CONCLUSIONS}

Overall, insights into the patients' perspective of living with NETs obtained from this survey have the potential to be valuable in the development of future information resources and support strategies that will complement currently available therapies to improve the experience and outcomes of patients living with NETs. The variety of responses indicate that a "one-size-fits-all" approach is not applicable in NETs, reflecting the heterogeneity of the population with NETs, as well as the disease itself, and that a tailored approach to symptom management, information, and support is required.

\section{ACKNOWLEDGEMENTS}

The authors wish to thank all the patients who responded to the survey, the patient and patient advocate organizations of APTED, CCF, and Netzwerk NeT e.V. for their collaboration, and Kay Wesley of Kanga Health for her assistance in setting up and conducting the survey.

Special thanks are owed to Katharina Mellar of Netzwerk NeT e.V., who was instrumental to this project and participated as an author on an earlier draft of this manuscript, but sadly died in August 2018.

Funding. This survey was sponsored by Ipsen, Boulogne Billancourt, France, which has also funded the journal's Rapid Service fee.

Medical Writing Assistance. Sarah Griffiths and Rebecca Crosby of Oxford PharmaGenesis and Richard Watt and Lindsay Queen of Sudler Medical Communications provided writing support, funded by Ipsen. 
Authorship. All named authors meet the International Committee of Medical Journal Editors criteria for authorship for this article, take responsibility for the integrity of the work as a whole, and have given their approval for this version to be published.

Authorship Contributions. All the authors contributed to the interpretation of the survey data and were involved in the process of writing and reviewing this manuscript. All authors take complete responsibility for the integrity of the data and accuracy of the data analysis. Grace Goldstein and Katharina Mellar helped to facilitate the survey, including review and approval of survey questions. Rupert Watts was instrumental in setting up and conducting the online survey itself, as well as providing survey data.

Disclosures. Mohid Khan has received speaker/consultancy fees and conference attendance fees from Ipsen and Novartis. Katharina Mellar: Netzwerk NeT e.V. received grants or sponsoring from Ipsen, Novartis, and Pfizer in 2016-given for other special projects and tasks, not for this study. Rupert Watts has received consultancy fees for Ipsen for NET (LivingwithNETs.com). Isabelle Bocher-Pianka is an employee of Ipsen with no other conflicts of interest to declare. Abdelali Majdi is an employee of Ipsen with no other conflicts of interest to declare. Grace Goldstein: the Carcinoid Cancer Foundation has received grants from Advanced Accelerator Applications, Ipsen, Lexicon, and Novartis.

Compliance with Ethics Guidelines. Respondents were aware of the purpose of the survey and gave their permission for their insights to be gathered in this voluntary process. Owing to the nature of the research as an insight-gathering exercise rather than as a clinical-grade piece of research, and the recruitment methods used, approval by an independent ethical committee was not required for this survey.

Data Availability. Where patient data can be anonymized, Ipsen will share all individual participant data that underlie the results reported in this article with qualified researchers who provide a valid research question. Study documents, such as the study protocol and clinical study report, are not always available. Proposals should be submitted to datasharing@ipsen.com and will be assessed by a scientific review board. Data are available beginning 6 months and ending 5 years after publication; after this time, only raw data may be available.

Open Access. This article is distributed under the terms of the Creative Commons Attribution-NonCommercial 4.0 International License (http://creativecommons.org/licenses/ by-nc/4.0/), which permits any noncommercial use, distribution, and reproduction in any medium, provided you give appropriate credit to the original author(s) and the source, provide a link to the Creative Commons license, and indicate if changes were made.

\section{REFERENCES}

1. Addario BJ, Fadich A, Fox J, Krebs L, Maskens D, Oliver $\mathrm{K}$, et al. Patient value: perspectives from the advocacy community. Health Expect. 2018;21(1): 57-63. https://doi.org/10.1111/hex.12628.

2. Yang LY, Manhas DS, Howard AF, Olson RA. Patientreported outcome use in oncology: a systematic review of the impact on patient-clinician communication. Support Care Cancer. 2018;26(1):41-60. https://doi.org/10.1007/s00520-017-3865-7.

3. Oronsky B, Ma PC, Morgensztern D, Carter CA. Nothing but NET: a review of neuroendocrine tumors and carcinomas. Neoplasia. 2017;19(12):991-1002. https://doi.org/10.1016/j.neo.2017.09.002.

4. Singh S, Granberg D, Wolin E, Warner R, Sissons M, Kolarova T, et al. Patient-reported burden of a neuroendocrine tumor (NET) diagnosis: results from the first global survey of patients with NETs. J Glob Oncol. 2016;3(1):43-53. https://doi.org/10.1200/ JGO.2015.002980.

5. Wolin EM, Leyden J, Goldstein G, Kolarova T, Hollander R, Warner RRP. Patient-reported experience of diagnosis, management, and burden of neuroendocrine tumors: results from a large patient survey in the United States. Pancreas. 2017;46(5):639-47. https://doi.org/10.1097/MPA.0000000000000818. 